\title{
Evolutionary and clinical trajectories of early-onset prostate cancer
}
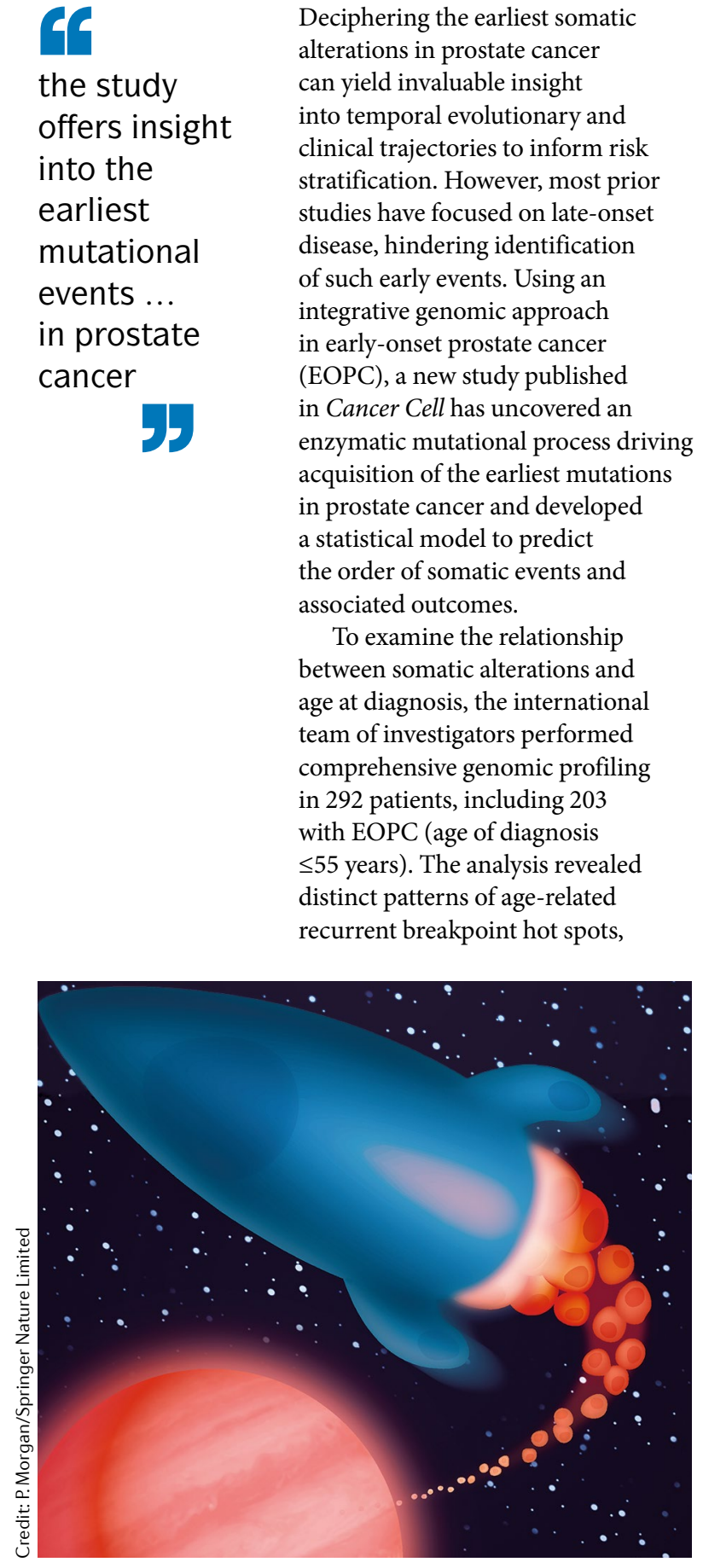

termed recurrent genomic alterations (RGAs), in EOPCs that were consistent with a primarily clonal origin, including a clinically relevant RGA at $8 \mathrm{q} 22$. The $8 \mathrm{q} 22$ region displayed recurrent genomic duplications of ESRP1, which encodes an RNA-binding protein involved in epithelial-mesenchymal transition and RNA splicing. Importantly, tissue microarray analysis $(n=11,954)$ revealed that high ESRP1 immunostaining correlated with high proliferation index, Gleason score, tumour stage, nodal metastasis burden and preoperative PSA levels, as well as short time to biochemical recurrence (BCR). Further multivariate analysis established ESRP1 as an independent prognostic marker.

Genome-wide analysis also revealed an APOBEC-associated mutational signature that exhibited a clock-like temporal pattern of mutation accumulation. APOBECassociated mutations were enriched at C/G (kataegis) clusters, which commonly lead to localized hypermutation. Moreover, kataegis events - primarily those attributable to APOBEC3 - were associated with patient age and frequently colocalized with breakpoints, including RGAs resulting in driver gene alterations. The findings suggest that APOBEC 3 drives an endogenous, age-associated mutagenic process responsible for clock-like accumulation of early somatic mutations in prostate cancer.

Next, methylation arrays and RNA sequencing were used to investigate cell type composition and gene expression patterns in EOPC. To this end, the authors developed and validated the purity-adjusted epigenetic prostate cancer index (PEPCI), a methylation-based score estimating the cell type composition and clinical aggressiveness of each tumour. Integrated analysis of PEPCI scores and CLICK cluster signatures - based on CLICK, a clustering algorithm for gene expression data with prognostic value - revealed four distinct molecular subgroups with distinct cell composition and transcriptional signatures that were associated with clinical outcome. Clinical validation in The Cancer Genome Atlas (TCGA) cohort $(n=462)$ showed that the subgroups could predict BCR in patients with intermediaterisk Gleason score 7, demonstrating prognostic potential.

The authors leveraged the enrichment for early somatic alterations and the high clonality of their EOPC cohort to develop PRESCIENT (prediction of sequential changes in the evolution of nascent tumours), a statistical model that uses molecular markers to predict the temporal order of somatic events and the associated probability of event-free survival. Importantly, PRESCIENT could predict outcomes and the next alteration in a cohort of 40 patients with local metastasis and was more accurate than frequency-based models.

Overall, the study offers insight into the earliest mutational events and evolutionary trajectories in prostate cancer and expertly demonstrates how such knowledge can be integrated into a framework for prognostication.

Conor A. Bradley

ORIGINAL ARTICLE Gerhauser, C. et al. Molecular evolution of early-onset prostate cancer identifies molecular risk markers and clinical trajectories. Cancer Cell 34, 996-1011 (2018) 